\title{
Left Ventricular Structure during Antihypertensive Treatment in Patients with Chronic Kidney Disease
}

\author{
Batir T. Daminov ${ }^{1}, \mathrm{PhD}, \mathrm{ScD}$; Sherzod S. Abdullaev²* \\ ${ }^{1}$ Tashkent Pediatric Medical Institute; ${ }^{2}$ Tashkent Medical Academy \\ Tashkent, Uzbekistan
}

\begin{abstract}
The aim of our study was to investigate the left ventricular (LV) echocardiographic parameters and estimate the antiremodeling efficacy of eprosartan and lercanidipine in patients with chronic kidney disease, depending on the presence or absence of diabetic nephropathy (DN).

Materials and Methods: The study included 121 patients (mean age 52.4 \pm 5.7 years) with CKD stage 3 (KDOQI, 2002). Patients were distributed in two groups according to the etiology of CKD. Group 1 consisted of 67 patients with non-diabetic CKD. Group 2 consisted of 54 CKD patients with DN. All patients had arterial hypertension grade 1 or 2 (ESH/ESC, 2013). All patients underwent clinical examination, echocardiography; GFR was estimated by the Cockcroft-Gault formula. Stages of chronic kidney disease (CKD) were determined according to the KDOQI 2002 classification. Eprosartan and lercanidipine were prescribed to patients after one week of lavage from previous antihypertensive therapy. This 6-month follow-up study compared the effectiveness of two courses of treatment.

Results: Left ventricular hypertrophy (LVH) was observed in all CKD patients regardless of the presence or absence of DN. Eprosartan and lercanidipine showed the high antihypertensive efficacy expressing a reliable decrease in absolute values of SBP and DBP. In CKD patients with DN, on the background of a comparable antihypertensive effect, eprosartan, in comparison with lercanidipine, showed a more pronounced effect on the LV echocardiographic parameters associated with LVH regression. (Int J Biomed. 2015;6(1):18-21.).
\end{abstract}

Keywords: chronic kidney disease; echo-geometric parameters; left ventricular hypertrophy; eprosartan; lercanidipine.

\section{Abbreviations}

LVEDD, left ventricular end-diastolic dimension; LVESD, left ventricular end-systolic dimension; LVEDV, left ventricular end-diastolic volume; LVESV, left ventricular end-systolic volume; LVSV, left ventricular stroke volume; IVST, interventricular septal thickness; LVPWT, left ventricular posterior wall thickness; RWT, relative wall thickness; LVM, left ventricular mass; LVMI, left ventricular mass index; LVH, left ventricular hypertrophy; GFR, glomerular filtration rate; CKD, chronic kidney disease; ESRD, end-stage renal disease.

\section{Introduction}

Cardiovascular disease (CVD) remains the major cause of death in patients with chronic kidney disease (CKD). According to a well-established classification, cardiovascular involvement in CKD can be set in the context of cardiorenal syndrome type 4 [1]. The National Kidney Foundation Task Force about CVD in CKD has emphasized the high risk of CVD in patients with CKD, and has identified LVH and

*Corresponding author: Sherzod S. Abdullaev. Tashkent Medical Academy. Tashkent, Uzbekistan. E-mail: dr.sherzod@ rambler.ru coronary artery disease as the major targets for intervention [2]. The prevalence of LVH is estimated to be between 16 and $31 \%$ in individuals with a GFR $>30 \mathrm{ml} / \mathrm{min}$; it increases to $60-75 \%$ prior to starting renal replacement therapy [3]. Foley et al. [4] followed 596 incident hemodialysis patients with no prior history of cardiac disease to investigate whether the incidence of LVH correlates with the duration of dialysis. After 18 months of dialysis, the author reported that $62 \%$ of the patients had an increased LV mass volume index and that $49 \%$ of them developed overt LV failure. These observations raise the question of whether dialysis therapy develops into LVH in ESRD patients [5]. The evaluation of LVH is a quite heterogeneous. Electrocardiography, 2D and 3D 
echocardiography $(\mathrm{ECHO})$ and cardiac magnetic resonance imaging (CMRI) represent three next steps to quantify and estimate the degree of LVH. Because of these clear limits of CMRI, ECHO is still established as the main device to evaluate LV mass in daily clinical practice although there are limitations in the determination and quantification of LVH [3].

Levi et al. [6] examined the relation of left ventricular mass to the incidence of cardiovascular disease, mortality from cardiovascular disease, and mortality from all causes in 3220 subjects enrolled in the Framingham Heart Study who were 40 years of age or older and free of clinically apparent cardiovascular disease, in whom left ventricular mass was determined echocardiographically. During a four-year followup period, there were 208 incident cardiovascular events, 37 deaths from cardiovascular disease, and 124 deaths from all causes. LVM, determined echocardiographically, was associated with all outcome events. This relation persisted after we adjusted for age, diastolic blood pressure, pulse pressure, treatment for hypertension, cigarette smoking, diabetes, obesity, the ratio of total cholesterol to high-density lipoprotein cholesterol, and electrocardiographic evidence of LVH. In men, the risk factor-adjusted relative risk of cardiovascular disease was 1.49 for each increment of $50 \mathrm{~g} / \mathrm{m}$ in LVM corrected for the subject's height (95\% CI: 1.20 to 1.85 ); in women, it was 1.57 (95\%CI: 1.20 to 2.04 ). LVM (corrected for height) was also associated with the incidence of death from cardiovascular disease ( $\mathrm{RR}=1.73$ [95\% CI: 1.19 to 2.52] in men and 2.12 [95\%CI: 1.28 to 3.49 ] in women). LVM (corrected for height) was associated with death from all causes ( $\mathrm{RR}=1.49$ [95\% CI: 1.14 to 1.94$]$ in men and 2.01 [95\%CI:1.44 to 2.81] in women). Authors concluded that the estimation of LVM by echocardiography offers prognostic information beyond that provided by the evaluation of traditional cardiovascular risk factors. An increase in LVM predicts a higher incidence of clinical events, including death, attributable to cardiovascular disease.

Similar results were obtained in other studies. The aim of the study performed by E.Paoletti et al. [ 7 ] was to identify patient- and haemodialysis -related specific factors that might be associated with a higher risk of sudden cardiac death in subjects receiving renal replacement treatment (RRT) and observed over 10 year period.. The study included 123 patients (76 men; age 29-79 years) undergoing RRT for at least 6 months. During the 10 years, 85 patients died -16 from SCD, 30 from cardiac causes (CC) other than SCD, and 39 from other causes. Univariate Cox regression analysis demonstrated that the factors increasing the risk of SCD were CHD $(P=0.002)$, the worsening of LVH $(P<0.0001)$, and the presence of long-lasting arterial hypertension $(P=0.001)$. An increase in LVH was the sole risk factor for SCD when comparing SCD with $\mathrm{CC}$ patients $(P=0.003)$. By multivariate Cox regression analysis $\triangle \mathrm{LVMI}$ was identified as the strongest predictor of SCD $(P<0.0001)$.

The severity and persistence of LVH are strongly associated with mortality risk and cardiovascular events in CKD and ESRD patients as reported by Zoccali et al. [8] and London et al. [9] who observed how a $10 \%$ decrease in LVM was translated into a $28 \%$ decrease in cardiovascular mortality risk in a cohort of patients on hemodialysis. The predictors of LVH regression include better control of systolic blood pressure $[10,11]$, a lower pulse wave velocity and higher hemoglobin levels [12]. It should be clear by now that more clinical trials are needed to assess guidelines for treating CKD-related LVH.

The aim of our study was to investigate the left ventricular echocardiographic parameters and estimate the antiremodeling efficacy of eprosartan and lercanidipine in CKD patients, depending on the presence or absence of DN.

\section{Materials and Methods}

The study included 121 patients (mean age 52.4 \pm 5.7 years) with CKD stage 3 . The stage of CKD was identified based on the level of kidney function, irrespective of diagnosis, according to the KDOQI CKD classification (2002) [14].

Patients were distributed in two groups according to the etiology of CKD. Group 1 consisted of 67 patients with nondiabetic CKD (49 patients with chronic glomerulonephritis, 15 patients with chronic pyelonephritis, and 3 patients with polycystic kidney disease). Group 2 consisted of 54 CKD patients with DN according to the criteria of the Committee on Diabetic Nephropathy [15]. All patients had arterial hypertension grade 1 or 2 (ESH/ESC, 2013). Exclusion criteria were CKD stages 4-5, coronary heart disease, atrial fibrillation and life-threatening ventricular arrhythmias, cancer, arterial hypertension (BP>159/99 $\mathrm{mmHg}$ ), chronic heart failure (NYHA FC>II), and patients receiving renal replacement therapy.

The control group consisted of 25 healthy, age-matched, randomly selected persons without clinical and instrumental signs of CKD.

Before and during treatment all patients were checked on office BP using Korotkov's method and ambulatory blood pressure monitoring.

Two-dimensional and M-mode echocardiography were done in accordance with American Society of Echocardiography recommendations using Toshiba SSH160A (Japan). The following parameters were measured and calculated: IVST, LVPWT, LVEDD, LVESD, LVEDV, LVESV, LVEF, and LVM. LVM was indexed to body surface area (LVMI). Left ventricular hypertrophy (LVH) was defined as LVMI of $>110 \mathrm{~g} / \mathrm{m}^{2}$ (women) and $>134 \mathrm{~g} / \mathrm{m}^{2}$ (men) [13].

GFR was estimated by the Cockcroft-Gault formula. Stages of chronic kidney disease (CKD) were determined according to the KDOQI 2002 classification.

Eprosartan and lercanidipine were prescribed to patients after one week of lavage from previous antihypertensive therapy. This 6-month follow-up study compared the effectiveness of two courses of treatment. Among Group 1 patients, 35 patients received eprosartan $(600 \mathrm{mg} /$ day $)$ and 32 patients, lercanidipine (10 mg/day). Among Group 2 patients, 28 patients received eprosartan $(600 \mathrm{mg} /$ day $)$ and 26 patients, lercanidipine (10 mg/day). Eprosartan and lercanidipine were prescribed in addition to standard treatment.

The study was conducted in accordance with ethical principles of the Declaration of Helsinki. It was approved by 
the Tashkent Medical Academy Ethics Committee. Written informed consent was obtained from all participants.

Statistical analysis was performed using the statistical software «Statistica». (v6.0, StatSoft, USA). Baseline characteristics were summarized as frequencies and percentages for categorical variables and as mean \pm SEM for continuous variables. Student's unpaired and paired t-tests were used to compare two groups for data with normal distribution. Group comparisons with respect to categorical variables are performed using chi-square tests or, alternatively, Fisher's exact test when expected cell counts were less than 5. A probability value of $P<0.05$ was considered statistically significant.

\section{Result and Discussion}

LVH was observed in all CKD patients regardless of the presence or absence of DN. Eprosartan and lercanidipine showed the high antihypertensive efficacy expressing a reliable decrease in absolute values of SBP and DBP in both groups.

In Group 1 patients, compared to the control group, LVEDD, LVEDV, LVESD and LVESV, as well as LVPWT and IVST were significantly $(P<0.05$ in all cases) greater (Table 1). In Group 2 patients, we found a more pronounced increase in LVEDD, LVEDV, LVESD, LVESV, LVPWT, and IVST $(P<0.01$ in all cases). Consequently, LV dilation was more pronounced in Group 2 patients. Changes in LVEF and LVSV in both groups were not significantly different from the control values.

\section{Table 1.}

Left ventricular echocardiographic parameters in CKD patients before therapy

\begin{tabular}{|l|c|c|c|}
\hline \multicolumn{1}{|c|}{ Parameters } & CG & Group 1 & Group 2 \\
\hline LVEDD, mm & $43.1 \pm 1.18$ & $48.1 \pm 3.44^{*}$ & $52.0 \pm 3.78^{*}$ \\
\hline LVESD, mm & $31.2 \pm 1.14$ & $38.8 \pm 3.26^{*}$ & $42.1 \pm 3.49^{* *}$ \\
\hline LVEDV, $\mathrm{ml}$ & $122.1 \pm 5.12$ & $148.4 \pm 8.43^{* * *}$ & $156.3 \pm 11.24^{* * *}$ \\
\hline LVESV, ml & $43.6 \pm 4.75$ & $55.3 \pm 6.43$ & $59.8 \pm 8.61^{*}$ \\
\hline LVSV, $\mathrm{ml}$ & $78.5 \pm 5.16$ & $92.1 \pm 6.05$ & $95.2 \pm 8.47$ \\
\hline LVEF, $\%$ & $64.3 \pm 4.12$ & $61.4 \pm 5.67$ & $60.3 \pm 6.34$ \\
\hline LVPWT, mm & $8.6 \pm 0.73$ & $11.5 \pm 1.25^{*}$ & $12.6 \pm 1.49^{* *}$ \\
\hline IVST, mm & $8.7 \pm 0.81$ & $11.7 \pm 1.43^{*}$ & $13.0 \pm 1.52^{* *}$ \\
\hline LVM, g & $124.9 \pm 15.36$ & $269.3 \pm 42.74^{* * *}$ & $283.4 \pm 48.36^{* * *}$ \\
\hline LVMI, g/m $\mathrm{m}^{2}$ & $70.2 \pm 11.21$ & $162.4 \pm 21.62^{* * *}$ & $169.5 \pm 27.75^{* * *}$ \\
\hline RWT \% & $0.35 \pm 0.05$ & $0.52 \pm 0.07^{*}$ & $0.55 \pm 0.09^{*}$ \\
\hline
\end{tabular}

$C G$ - control group; * $-P<0.05$; ** $-P<0.01$; *** $-P<0.001$ versus the control group.

At the same time, we found a significant increase in LVM, LVMI and RTW, especially in Group 2 patients. Thus, in Group 1 patients LVM, LVMI and RTW exceeded the normative values by $2.15(P<0.001), 2.31(P<0.001)$ and $1.48(P<0.05)$ times, respectively, and in Group 2 patients by $2.27(P<0.001), 2.41(P<0.001)$ and $1.57(P<0.05)$ times, respectively.

The changes in LV echocardiographic parameters during the 6-month therapy with eprosartan and lercanidipine are shown in Table 2. Eprosartan effects were more pronounced than lercanidipine effects in Group 1 patients with non-diabetic CKD. For example, we found a marked reduction of LVEDD, LVESD, LVEDV, LVESV, LVPWT, and IVST during treatment with eprosartan compared to lercanidipine; however, the values of these parameters were not fully normalized compared to the control group. LVSV tended to decrease and LVEF to increase compared to initial values. Initially high values of LVM, LVMI and RTW in these patients significantly decreased after the 6-month treatment with eprosartan $(P<0.05$ in all cases). In particular, in Group 1 patients, a 6-month therapy with eprosartan was associated with significant decrease in LVMI by $17.8 \%$ versus $11.6 \%$ during therapy with lercanidipine $(P<0.05)$.

Table 2.

Left ventricular echocardiographic parameters in CKD patients after 6-month treatment with Eprosartan and lercanidipine

\begin{tabular}{|l|c|c|c|c|}
\hline \multirow{2}{*}{ Parameters } & \multicolumn{2}{|c|}{ Group 1 } & \multicolumn{2}{c|}{ Group 2 } \\
\cline { 2 - 5 } & LER & EPR & LER & EPR \\
\hline LVEDD, mm & $43.82 \pm 3.32$ & $42.3 \pm 2.13^{*}$ & $48.7 \pm 3.27$ & $47.3 \pm 2.75^{*}$ \\
\hline LVESD, $\mathrm{mm}$ & $35.1 \pm 3.18$ & $33,6 \pm 2.15^{*}$ & $38.8 \pm 3.09$ & $37.4 \pm 2.41^{*}$ \\
\hline LVEDV, $\mathrm{ml}$ & $133.1 \pm 8.55$ & $127.3 \pm 8.22 *$ & $141.2 \pm 11.56$ & $135.2 \pm 10.22^{*}$ \\
\hline LVESV, $\mathrm{ml}$ & $46.5 \pm 6.35$ & $41.5 \pm 6.28^{*}$ & $52.7 \pm 7.34$ & $47.6 \pm 6.53^{*}$ \\
\hline LVSV, $\mathrm{ml}$ & $86.6 \pm 6.32$ & $85.7 \pm 6.17$ & $88.5 \pm 7.45$ & $86.6 \pm 7.23$ \\
\hline LVEF, \% & $65.1 \pm 5.67$ & $67.4 \pm 5.87$ & $62.5 \pm 5.72$ & $64.2 \pm 6.32$ \\
\hline LVPWT, mm & $10.7 \pm 1.23$ & $10.3 \pm 1.12 *$ & $11.9 \pm 1.44$ & $11.5 \pm 1.16^{*}$ \\
\hline IVST, $\mathrm{mm}$ & $10.9 \pm 1.28$ & $10.5 \pm 1.18^{*}$ & $12.2 \pm 1.35$ & $11.9 \pm 1.12^{*}$ \\
\hline LVM, g & $234.6 \pm 42.23$ & $221.3 \pm 40.12 *$ & $248.2 \pm 44.63$ & $234.3 \pm 42.28^{*}$ \\
\hline LVMI, g/m ${ }^{2}$ & $143.6 \pm 21.76$ & $133.5 \pm 20.45^{*}$ & $150.7 \pm 25.47$ & $141.5 \pm 23.16^{*}$ \\
\hline RTW, \% & $0.50 \pm 0.06$ & $0.47 \pm 0.05^{*}$ & $0.52 \pm 0.07$ & $0.51 \pm 0,05^{*}$ \\
\hline
\end{tabular}

LER-Lercanidipine; EPR-Eprosartan; * $-P<0.05$ versus the indices before treatment.

In Group 2 patients with $\mathrm{DN}$ and $\mathrm{CKD}$, on the background of a comparable antihypertensive effect, lercanidipine, in comparison with eprosartan, did not show an appreciable effect on the LV parameters. All the studied parameters had only a tendency to normalization and were significantly different from the normative values.

In Group 2 patients, we found a reduction of LVEDD, LVESD, LVEDV, LVESV, LVPWT, and IVST during treatment with eprosartan compared to initial levels $(\mathrm{P}<0.05$ in all cases); however, the values of these parameters were not fully normalized compared to the control group. LVSV tended to decrease and LV EF to increase compared to initial values. Initially high values of LVM, LVMI and RTW in these patients significantly decreased after the 6-month treatment with eprosartan $(P<0.05$ in all cases), but these changes were less pronounced than in Group 1 patients treated with eprosartan, and not fully normalized.

In conclusion, it can be noted that the LV echo-geometric parameters in CKD patients with and without DN during longterm treatment with eprosartan and lercanidipine improved slightly and that the reverse remodeling was less pronounced in CKD patients with diabetic nephropathy. More pronounced changes were observed during treatment with eprosartan. However, we did not find a full normalization of the studied 
echo parameters, which indicates the preservation of LVH in the treated patients.

\section{Findings:}

1. LVH was observed in all CKD patients regardless of the presence or absence of DN.

2. In CKD patients with $\mathrm{DN}$, on the background of a comparable antihypertensive effect, eprosartan, in comparison with lercanidipine, showed a more pronounced effect on the LV echocardiographic parameters associated with LVH regression.

\section{Competing interests}

The authors declare that they have no competing interests.

\section{References}

1. House AA, Anand I, Bellomo R, Cruz D, Bobek I, Anker SD, et al.; Acute Dialysis Quality Initiative Consensus Group. Definition and classification of Cardio-Renal Syndromes: workgroup statements from the 7th ADQI Consensus Conference. Nephrol Dial Transplant. 2010;25(5):1416-20.

2. Meyer KB, Levey AS. Controlling the epidemic of cardiovascular disease in chronic renal disease: Report from the National Kidney Foundation Task Force on Cardiovascular Disease. J Am Soc Nephrol. 1998; 9(12 Suppl):S31- S42.

3. Di Lullo L, Gorini A, Russo D, Santoboni A, Ronco C. Left Ventricular Hypertrophy in Chronic Kidney Disease Patients: From Pathophysiology to Treatment. Cardiorenal Med. 2015;5:254-266

4. Foley RN, Curtis BM, Randell EW, Parfrey PS. Left ventricular hypertrophy in new hemodialysis patients without symptomatic cardiac disease. Clin J Am Soc Nephrol. 2010;5(5):805-13.

5. Parikh SV, de Lemos JA. Biomarkers in cardiovascular disease: integrating pathophysiology into clinical practice. Am J Med Sci. 2006;332(4):186-97.

6. Levy D, Garrison RJ, Savage DD, Kannel WB, Castelli WP. Prognostic implications of echocardiographically determined left ventricular mass in the Framingham Heart Study. N Engl J Med. 1990;322(22):1561-6.

7. Paoletti E, Specchia C, Di Maio G, Bellino D, Damasio B, Cassottana P, Cannella G. The worsening of left ventricular hypertrophy is the strongest predictor of sudden cardiac death in haemodialysis patients: a 10 year survey. Nephrol Dial Transplant. 2004;19(7):1829-34.

8. Zoccali C, Benedetto FA, Mallamaci F, Tripepi G, Giacone G, Stancanelli B, et al. Left ventricular mass monitoring in the follow-up of dialysis patients: prognostic value of left ventricular hypertrophy progression. Kidney Int. 2004;65(4):1492-8.

9. London GM, Pannier B, Guerin AP, Blacher J, Marchais SJ, Darne B, et al. Alterations of left ventricular hypertrophy in and survival of patients receiving hemodialysis: follow-up of an interventional study. J Am Soc Nephrol. 2001;12(12):2759 -67. 10. Mattioli AV, Zennaro M, Bonatti S, Bonetti L, Mattioli G. Regression of left ventricular hypertrophy and improvement of diastolic function in hypertensive patients treated with telmisartan. Int J Cardiol. 2004;97(3):383-8.

11. Devereux RB, Palmieri V, Liu JE, Wachtell K, Bella JN, Boman K, et al. Progressive hypertrophy regression with sustained pressure reduction in hypertension: the Losartan Intervention for Endpoint Reduction study. J Hypertens. 2002;20(7):1445-50.

12. Krane V, Winkler K, Drechsler C, Lilienthal J, Marz W, Wanner C; German Diabetes and Dialysis Study Investigators. Effect of atorvastatin on inflammation and outcome in patients with type 2 diabetes mellitus on hemodialysis. Kidney Int. 2008;74(11):1461-7.

13. Abergel E, Tase M, Bohlender J, Menard J, Chatellier G. Which definition for echocardiographic left ventricular hypertrophy? Am J Cardiol. 1995; 75(7):498-502.

14. K/DOQI clinical practice guidelines for chronic kidney disease: evaluation, classification, and stratification. National Kidney Foundation. Am J Kidney Dis. 2002;39(2 Suppl 1):S1S266.

15. Haneda M, Utsunomiya K, Koya D, Babazono T, Moriya T, Makino H, et al.; Joint Committee on Diabetic Nephropathy. A new Classification of Diabetic Nephropathy 2014: a report from Joint Committee on Diabetic Nephropathy. J Diabetes Investig. 2015;6(2):242-6. 